\title{
Flat Bands and Giant Light-Matter Interaction in Hexagonal Boron Nitride
}

\author{
C. Elias $\odot,{ }^{1}$ G. Fugallo, ${ }^{2}$ P. Valvin $\odot,{ }^{1}$ C. L'Henoret, ${ }^{1}$ J. Li® ${ }^{3}$ J. H. Edgar, ${ }^{3}$ F. Sottile, ${ }^{4}$ \\ M. Lazzeri $\odot,{ }^{5}$ A. Ouerghi, ${ }^{6}$ B. Gil, ${ }^{1}$ and G. Cassabois $\circledast^{1, *}$ \\ ${ }^{1}$ Laboratoire Charles Coulomb UMR 5221 CNRS-Université de Montpellier, 34095 Montpellier, France \\ ${ }^{2}$ LTeN UMR 6607 CNRS-PolytechNantes, Université de Nantes, 44306 Nantes, France \\ ${ }^{3}$ Tim Taylor Department of Chemical Engineering, Kansas State University, Manhattan, Kansas 66506, USA \\ ${ }^{4}$ Laboratoire des Solides Irradiés, Ecole Polytechnique, CNRS, CEA/DRF/IRAMIS, Institut Polytechnique de Paris, \\ 91128 Palaiseau, France \\ ${ }^{5}$ Sorbonne Université, CNRS UMR 7590, MNHN, IMPMC, 75005 Paris, France \\ ${ }^{6}$ C2N CNRS-Univ Paris Sud, Université Paris-Saclay, 91120 Palaiseau, France
}

(Received 24 November 2020; accepted 31 August 2021; published 23 September 2021)

\begin{abstract}
Dispersionless energy bands in $k$ space are a peculiar property gathering increasing attention for the emergence of novel electronic, magnetic, and photonic properties. Here, we explore the impact of electronic flat bands on the light-matter interaction. The van der Waals interaction between the atomic layers of hexagonal boron nitride induces flat bands along specific lines of the Brillouin zone. The macroscopic degeneracy along these lines leads to van Hove singularities with divergent joint density of states, resulting in outstanding optical properties of the excitonic states. For the direct exciton, we report a giant oscillator strength with a longitudinal-transverse splitting of $420 \mathrm{meV}$, a record value, confirmed by our $a b$ initio calculations. For the fundamental indirect exciton, flat bands result in phonon-assisted processes of exceptional efficiency, that compete with direct absorption in reflectivity, and that make the internal quantum efficiency close to values typical of direct band gap semiconductors.
\end{abstract}

DOI: $10.1103 /$ PhysRevLett.127.137401

Flat-band engineering is the subject of intense research for investigating strongly correlated states in systems made of electrons, cold atoms, and photons [1]. From a curiosity in magnetic frustration, flat bands have become a key fingerprint sought for in synthetic devices for studying superconductivity arising from electronic interactions, correlated insulator states, spontaneous ferromagnetism and the quantum Hall effect [2-4]. Flat bands exist naturally in materials such as bulk van der Waals insulators, hexagonal boron nitride $(\mathrm{hBN})$ being a model example. With the advent of graphene and transition metal dichalcogenides, $\mathrm{hBN}$ has become a key compound in 2D materials research, with the usual indirect-direct crossover of the band gap at the monolayer limit [5-8]. However, there is also a rich physics in the bulk form of hBN, with original photonic properties that have been only recently explored [9].

In this Letter, we report giant light-matter interaction arising from electronic flat bands in bulk hBN. In addition to the weak interlayer interaction typical of van der Waals structures, the ionicity difference between $\mathrm{B}$ and $\mathrm{N}$ enhances localization effects, resulting in electronic flat bands along specific Brillouin zone lines parallel to the $c$ axis. The macroscopic degeneracy along these lines leads to van Hove singularities with divergent joint density of states, resulting in unique optoelectronic signatures. The combination of reflectivity and photoluminescence (PL) experiments in state-of-the-art hBN crystals allows us to characterize direct and indirect excitons and compare our results to a phenomenological model and first-principles calculations. For the direct exciton, we report a giant oscillator strength with a longitudinal-transverse splitting of $420 \mathrm{meV}$, the highest value ever reported. For the fundamental indirect exciton, flat bands translate in highly efficient phonon-assisted processes, which compete with direct absorption in reflectivity, and that make the internal quantum efficiency close to values typical of direct band gap semiconductors.

Bulk $\mathrm{hBN}$ is a layered material considered the insulating analog of graphite and known as "white graphite." The fundamental band gap of $\sim 6 \mathrm{eV}$ is indirect in bulk hBN with a minimum of the conduction band at the $M$ point and a maximum of the valence band around K [Fig. 1(a)]. There is a direct band gap at the $K$ point [Fig. 1(a)], slightly higher in energy than the indirect one. Excitonic effects are huge in $\mathrm{hBN}$ with a binding energy of $\sim 0.7 \mathrm{eV}$ [10-12]. In order to take into account the electron-hole interaction in the evaluation of the dielectric function $\epsilon_{\mathbf{q}, \omega}$ one has to solve the Bethe-Salpeter equation [13]. The solution is most often written in a basis of independent-particle transitions $t$ of oscillator strengths $\rho$ mixed by the exciton wave functions $A_{t}^{\lambda}$ :

$$
\epsilon_{\mathbf{q}, \omega} \propto \sum_{\lambda} \frac{\left|\sum_{t} A_{\lambda}^{t} \rho^{t}(\mathbf{q})\right|^{2}}{\hbar \omega-E_{\lambda}+i \eta}
$$



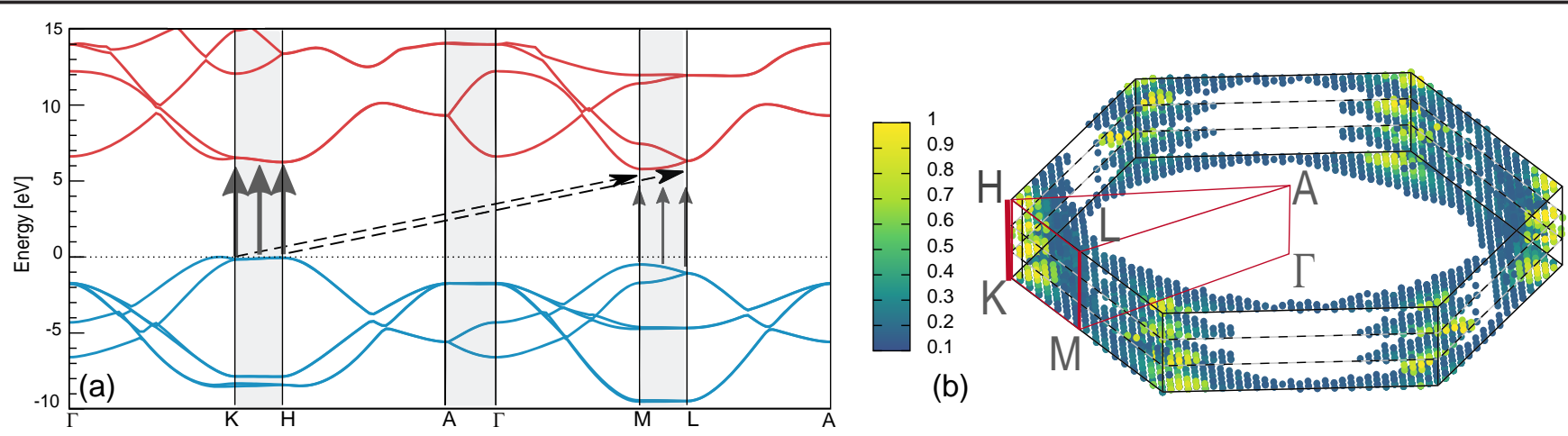

FIG. 1. (a) Electronic band structure evaluated in the framework of many-body GW calculations. The shaded areas indicate the paths that are parallel to the c-axis in the Brillouin zone of bulk hBN. Arrows in dark gray (dashed black) indicate the parallel direct (indirect) transitions contributing to the formation of the direct $(d X)$ and indirect $(i X)$ excitons, respectively. (b) Color map in the Brillouin zone of bulk hBN of the weighted oscillator strengths $A_{\lambda}^{t} \rho^{t}(\mathbf{q})$ [as defined in Eq. (1)] building the $d X$ exciton. For the sake of clarity, the map is limited to the points of the prevailing direct independent-particle transitions $t$, that contribute in total to $80 \%$ of the $d X$ absorption. The irreducible part of the Brillouin zone is delimited by red lines, which are thicker for the KH and ML paths.

where $t=v c$ labels a transition between a valence state $v$ and a conduction one $c$ and the sum runs over the $\lambda$ excitons with wave vector q (see Supplemental Material [14], Sec. ab initio calculations). In the independent-particle approximation (IPA), the dielectric function is given by the following simple expression:

$$
\epsilon_{\mathbf{q}, \omega}^{(\mathrm{IPA})} \propto \sum_{t} \frac{\left|\rho^{t}(\mathbf{q})\right|^{2}}{\hbar \omega-E_{t}+i \eta} .
$$

In the absence of electron-hole interaction [Eq. (2)], the optical resonances are governed, in a first approximation, by the optical matrix elements and the joint density of states. In the case of flat bands, the joint density of states presents van Hove singularities that stem from the large number of transitions contributing in parallel to a given optical resonance.

With the electron-hole interaction [Eq. (1)], the dielectric function is not only influenced by the van Hove singularities due to flat bands but also by the formation of the excitonic states. The products $A_{\lambda}^{t} \rho^{t}(\mathbf{q})$ can be called "weighted oscillator strengths" and the total intensity of the exciton peak is determined by the constructive coherent superposition of these terms [Eq. (1)]. By examining the first-order correction to the $A_{t}^{\lambda}$ wave functions: $A_{t}^{\lambda} \sim \sum_{t^{\prime}}\left[W_{t}^{t^{\prime}} /\left(E_{t}-E_{t^{\prime}}\right)\right]$, one observes that large terms arise from independent-particle transitions where the energy difference $E_{t}-E_{t^{\prime}}$ is small. Excitonic effects are therefore enhanced when mixing many independent-particle transitions with similar energy [40]. Besides the existence of van Hove singularities, this phenomenon further contributes to the emergence of outstanding optical properties in systems with flat bands.

The vertical and tilted arrows in Fig. 1(a) indicate all the transitions contributing to the formation of the direct $(d X)$ and indirect $(i X)$ excitons in $\mathrm{hBN}$, respectively $[7,11,41]$.
These independent-particle transitions come from regions of the Brillouin zone, parallel to the $c$ axis, where the valence and conduction bands are almost flat and parallel. The $i X$ exciton results from indirect transitions moving in parallel along the $c$ axis from $K \rightarrow M$ to $H \rightarrow L$ [Fig. 1(a)]. The $d X$ exciton builds as a collective excitation of the crystal involving all direct transitions along the $\mathrm{KH}$ and $\mathrm{ML}$ lines [Fig. 1(a)], as discussed in Refs. [11,42]. However, the most intense transitions [depicted in yellow in Fig. 1(b)] come from points distributed around the KH line. It reveals the contributions coming from the ML region are in fact negligible with respect to $\mathrm{KH}$ where the bands are flatter.

We have performed measurements by reflectivity and PL in high-quality crystals. Because of the indirect band gap of bulk hBN, PL in hBN probes the fundamental indirect exciton $i X$ which has an energy $E_{i X}=5.955 \mathrm{eV}$ [7]. The direct excitonic state $d X$ lies at a higher energy than $E_{i X}$ and it is expected to determine the optical response above the band gap of $\mathrm{hBN}$. The interplay between direct and indirect excitons in the optical properties of $\mathrm{hBN}$ remains an open issue because reflectivity and absorption experiments have been so far very limited in hBN. In the eighties, Hoffman et al. reported the first reflectivity measurements in $\mathrm{hBN}$, from the midinfrared to the deep ultraviolet, but at room temperature and in low-quality crystals made of pyrolytic boron nitride [43]. Watanabe et al. extended the experiments to high-quality crystals $[44,45]$. Although these measurements were performed at cryogenic temperatures, no significant advance followed in the interpretation of the complex excitonic structure close to the band gap of hBN. Here, we have achieved reflectivity experiments at low temperature with a high spectral resolution, for samples fabricated by a process enabling the formation of large single crystals [Fig. 2, inset] with a thickness of few micrometers (see Supplemental Material [14]). This experimental achievement has allowed us to reveal the fine structure of the reflectivity spectrum around $6 \mathrm{eV}$. 


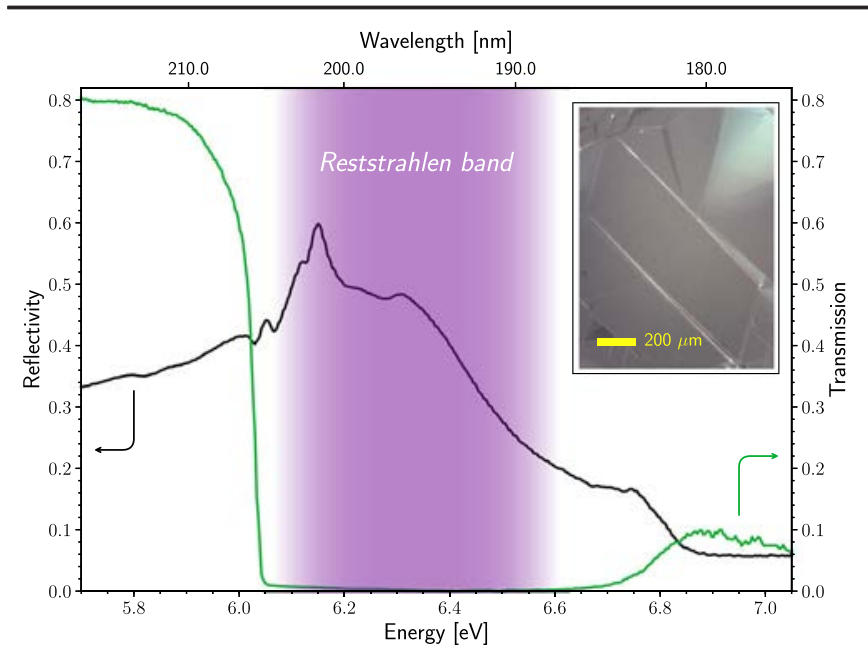

FIG. 2. Reflectivity (black) and transmission (green) of a highquality hBN crystal, recorded close to normal incidence at $8 \mathrm{~K}$. The crystal thickness is $4.5 \mu \mathrm{m}$. The shaded area indicates the excitonic reststrahlen band in $\mathrm{hBN}$. Inset: microscope image of high-quality hBN synthesized by the metal flux method.

We provide a quantitative interpretation of our reflectivity and PL data and we show how electronic flat bands result in exceptional optical properties of the direct and indirect excitons in hBN.

Reflectivity and transmission spectra in a $4.5 \mu \mathrm{m}$-thick hBN crystal at $8 \mathrm{~K}$ are plotted in Fig. 2. The transmission drops abruptly around $6 \mathrm{eV}$ and increases above noise level for energies larger than $6.6 \mathrm{eV}$. Such a spectrum is characteristic of the presence of a strong resonance above $6 \mathrm{eV}$. The spectral range of low transmission is the so-called reststrahlen band, the general terminology used for vibrational and electronic resonances in solid state [46]. Although the present crystal is still too thick to extract the absorption spectrum in this energy range, the transmission spectrum in Fig. 2 provides the spectral position of the excitonic reststrahlen band in $\mathrm{hBN}$. We point out an unusually large value of its bandwidth of the order of half an $\mathrm{eV}$, to be compared to few or tens of $\mathrm{meV}$ in standard semiconductors [46]. This is a first indication for the giant light-matter interaction and the existence of extremely large oscillator strengths in hBN. The reflectivity spectrum in Fig. 2 displays previously unresolved fine structures. In particular, our measurements reveal the existence of four relative maxima of the reflectivity at $6.02,6.05,6.11$, and $6.14 \mathrm{eV}$. The comparison with the PL spectrum in Fig. 3 will show that they correspond to phonon-assisted optical transitions by the fundamental indirect exciton lying at $E_{i X}=5.955 \mathrm{eV}$. The observation of phonon-assisted absorption lines in the reflectivity spectrum of an indirect semiconductor is a unique feature without any equivalent example in the literature [46]. This is a second indication for the exceptional efficiency of the light-matter interaction in $\mathrm{hBN}$, also stemming from the existence of electronic flat bands.

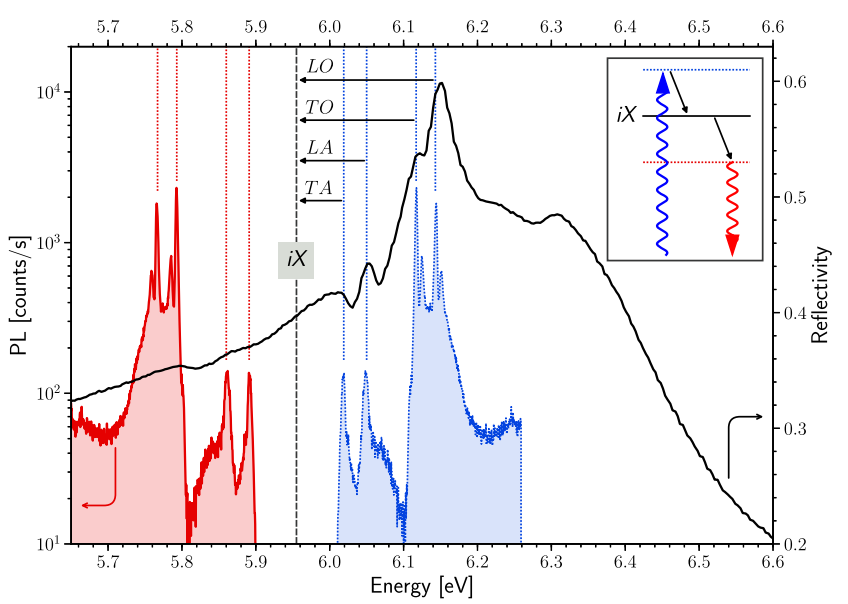

FIG. 3. Comparison between reflectivity (black) and photoluminescence (red) of bulk hBN at $8 \mathrm{~K}$. The vertical dashed line at $5.955 \mathrm{eV}$ indicates the energy of the indirect exciton $(i X)$. The dotted blue spectrum corresponds to the mirror image of the PL spectrum with respect to the $i X$ energy. Inset: schematic representation of absorption (blue) and emission (red) assisted by phonon emission (black arrow). The blue and red dashed lines indicate the virtual states involved in the phonon-assisted optical processes. $T A, L A, T O$, and $L O$ indicate the modes of the emitted phonons in reflectivity.

The indirect nature of the four lines detected in the reflectivity spectrum is demonstrated in Fig. 3 where PL data are superimposed to the reflectivity spectrum. More precisely, the PL spectrum is displayed in solid red line while its mirror image with respect to the $i X$ energy is shown in dotted blue line. Such a plot is motivated by the mirror symmetry between the processes of absorption and emission assisted by phonon emission in indirect semiconductors [46]: compared with the band gap energy, photon emission is redshifted by the phonon energy, while photon absorption is blueshifted by the same value [as shown in the inset of Fig. 3]. The comparison between the reflectivity spectrum and the mirrored PL one indicates that the four lines revealed by our reflectivity measurements are perfectly in resonance with the transitions due to phonon-assisted absorption in hBN. From the assignment by PL spectroscopy [7] and in agreement with polarization selection rules [47] the lines at 6.02, $6.05,6.11$, and $6.14 \mathrm{eV}$ correspond to optical absorption assisted by the emission of one $T A, L A, T O$, and $L O$ phonon, respectively [Fig. 3]. Such a phenomenology is in strong contrast to the textbook examples of phonon-assisted absorption. In standard semiconductors, indirect transitions appear as weak absorption bands where onset is difficult to identify $[46,48,49]$, requiring either data postprocessing [50] or modulation spectroscopy [46,51]. In hBN, phonon-assisted absorption is readily observed with high contrast in reflectivity experiments, a unique situation among indirect semiconductors. We note that the existence of narrow lines rather than broad bands stems from the strong $k$ dependence of the exciton-phonon matrix element in hBN [7]. 

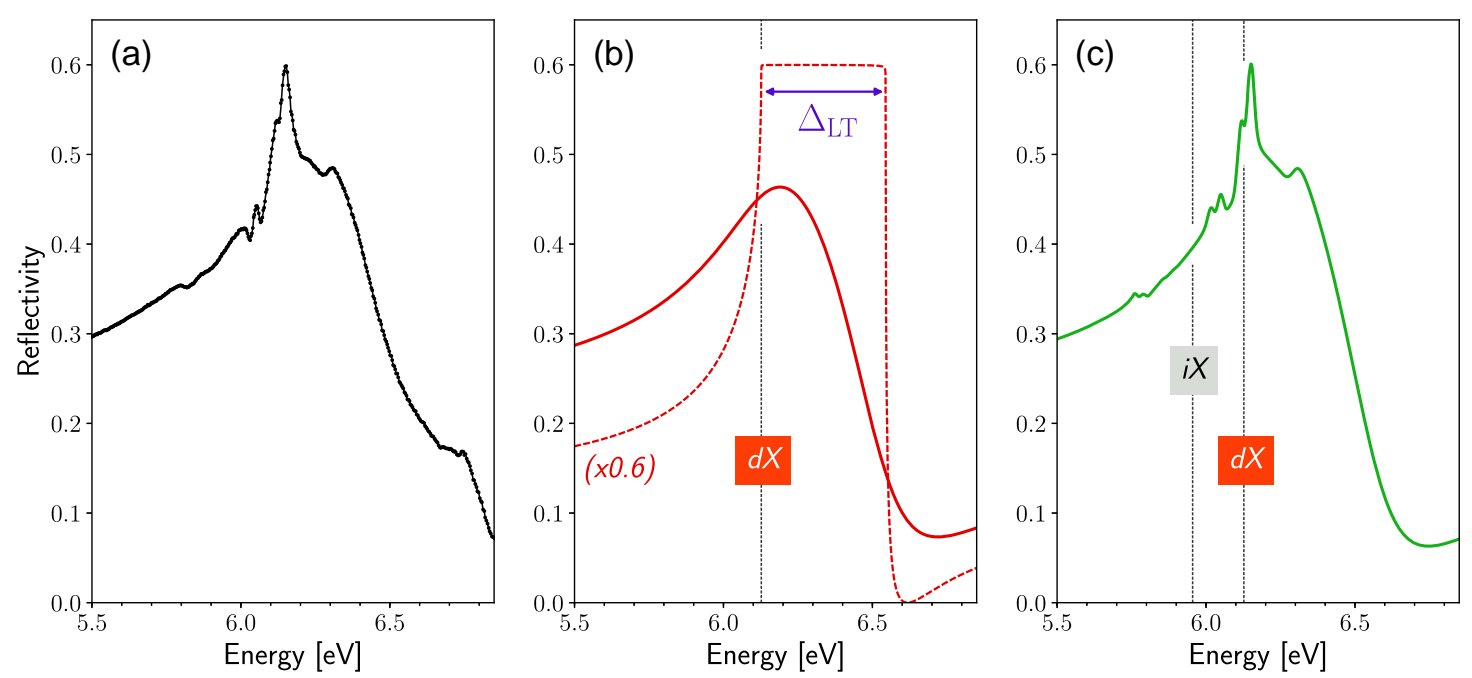

FIG. 4. (a) Experimental reflectivity spectrum at $8 \mathrm{~K}$. (b) Calculated reflectivity spectrum (red solid line) taking into account the direct exciton $(d X)$ only, with $\gamma / \Delta_{\mathrm{LT}}=0.41$, where $\gamma$ is the $d X$ linewidth and $\Delta_{\mathrm{LT}}$ is the longitudinal-transverse splitting. $\Delta_{\mathrm{LT}}=420 \mathrm{meV}$ in $\mathrm{hBN}$. The red dashed line is the reflectivity spectrum calculated for $\gamma / \Delta_{\mathrm{LT}}=10^{-4}$ in order to better visualize $\Delta_{\mathrm{LT}}$, which corresponds to the width of the reflectivity stop band when $\gamma / \Delta_{\mathrm{LT}} \ll 1$. (c) Calculated reflectivity spectrum (green solid line) taking into account the indirect $(i X)$ and direct $(d X)$ excitons $\left(\gamma / \Delta_{\mathrm{LT}}=0.41\right) . E_{i X}=5.955$ and $E_{d X}=6.125 \mathrm{eV}$.

The experimental reflectivity spectrum [Fig. 4(a)] is compared to the theoretical one calculated either with the optical transition of the direct exciton $d X$ only [Fig. 4(b)], or with both $d X$ and $i X$ contributions [Fig. 4(c)] (see Supplemental Material [14], Sec. "reflectivity modelling" for details on reflectivity calculations). The excellent agreement of our data with the calculated reflectivity spectrum in Fig. 4(c) shows that we have reached a quantitative interpretation of the optoelectronic properties of $\mathrm{hBN}$ close to the band gap. The direct exciton $d X$ gives a broad pedestal fixing the order of magnitude of the reflectivity to $\sim 50 \%$ around $6 \mathrm{eV}$ [Fig. 4(b)]. The indirect exciton $i X$ accounts for a series of narrow lines, that are phonon replicas decorating the smooth band of the direct exciton [Fig. 4(c)]. We highlight the spectral coincidence of direct and indirect optical transitions in the near band gap absorption of hBN. This peculiarity has so far hindered the comprehensive understanding of hBN physics [44,52]. Our results unravel the complex interplay between direct and indirect processes in $\mathrm{hBN}$, resulting in an unconventional optical response where the prominent peak at $6.1 \mathrm{eV}$ does not arise from the direct exciton $d X$ but from the indirect one $i X$.

From the quantitative interpretation of our experiments, we first comment on the giant oscillator strength of the direct exciton $d X$ due to electronic flat bands in $\mathrm{hBN}$. For a given value of the excitonic linewidth, the higher the oscillator strength, the higher the reflectivity [53]. Conversely, for a given oscillator strength, the smaller the excitonic linewidth, the higher the reflectivity, as shown by the dashed line in Fig. 4(b). In this limit, the reflectivity is of order unity in a spectral window corresponding to the so-called reststrahlen band. In complete analogy with optical phonons, the width of the excitonic restrahlen band is given by the energy difference $\Delta_{\mathrm{LT}}$ between transverse and longitudinal exciton states $[54,55]$. The longitudinaltransverse splitting $\Delta_{\mathrm{LT}}$ originates from Coulomb exchange interaction and it was shown that this exchange parameter can be remarkably expressed in a simple form as a function of the dipolar interaction matrix element $[54,55]$. Therefore $\Delta_{\mathrm{LT}}$ is a common metric of the efficiency of the light-matter interaction. In $\mathrm{hBN}$, we find $\Delta_{\mathrm{LT}}=420 \mathrm{meV}$ [Fig. 4], the highest value ever reported in the literature to the best of our knowledge (see Supplemental Table 1 in the Supplemental Material [14]). Instructive is a first comparison with AlN. AlN shares many similarities with hBN but the lamellar structure. Both are nitride semiconductors with a similar band gap of $\sim 6 \mathrm{eV}$, still $\Delta_{\mathrm{LT}}$ has a much lower value of $6 \mathrm{meV}$ in AlN [56]. In $\mathrm{hBN}, \Delta_{\mathrm{LT}}$ is enhanced by almost 2 orders of magnitude, a striking consequence of electronic flat bands. High values of $\Delta_{\mathrm{LT}}$ were reported in crystals with strongly bound excitons such as alkali halides [57,58], solid neon, and solid argon [59]. In these crystals, $\Delta_{\mathrm{LT}}$ follows the general scaling of the oscillator strength, that increases with the excitonic binding energy, and that is inversely proportional to the cube of the excitonic Bohr radius in the approximation of parabolic bands [60]. Usually, the more strongly bound the exciton is, the higher $\Delta_{\mathrm{LT}}[53,56]$. This standard rule in solid state is broken down because of the existence of flat bands. hBN provides an example of this effect, with exceptional degree as $\Delta_{\mathrm{LT}}$ surpasses any reported value in the literature, in particular the $360 \mathrm{meV}$ in solid argon [59] where the band gap is $\sim 14 \mathrm{eV}$. This means the macroscopic degeneracy of the direct transitions along the KH line parallel to the $c$ axis [Fig. 1(a)] has a dramatic impact on the optical properties of excitons in hBN, as illustrated by the ultralarge excitonic 
restrahlen band [Fig. 2] and the corresponding record value of $\Delta_{\mathrm{LT}}$ for the direct exciton $d X$ [Fig. 4(b)], confirmed by our first-principles calculations (see Supplemental Material [14], Sec. ab initio calculations).

The light-matter interaction for the indirect exciton $i X$ is radically different because of the coupling to the phonons field for satisfying the $k$-selection rule. Still, the parallel indirect transitions between the flat bands along the $\mathrm{KH}$ and ML lines [Fig. 1(a)] play a key role in the optical properties of $i X$. From the quantitative interpretation of our data [Fig. 4], one can analyze the effective oscillator strength of the phonon replicas identified from the comparison between PL and reflectivity (Fig. 3). For the optical phonon replicas ( $T O$ and $L O$ lines), the effective oscillator strength is 20 times smaller than for $d X$ (see Supplemental Material [14], Sec. reflectivity modelling for details on reflectivity calculations). Such a reduction stems from the higher order of phonon-assisted optical transitions involving dipolar interaction and electron-phonon coupling. Still, the $T O$ and $L O$ replicas in $\mathrm{hBN}$ have an oscillator strength equivalent to direct materials with a longitudinal-transverse splitting of order $20 \mathrm{meV}$. The latter value is higher than $\Delta_{\mathrm{LT}}$ in direct wide band gap semiconductors such as GaN, AlN, and $\mathrm{ZnO}$, where $\Delta_{\mathrm{LT}}=0.8,6$, and $11 \mathrm{meV}$, respectively [56]. This means electronic flat bands in $\mathrm{hBN}$ make phonon-assisted optical transitions stronger than direct ones in materials used in the optoelectronics industry $[61,62]$. It also shows that the outstanding properties of indirect transitions in $\mathrm{hBN}$ are intrinsic, readily imprinted in the dielectric constant, and observable in reflectivity and absorption experiments. As a consequence, this phenomenon also contributes to reaching an internal quantum efficieny of order unity in hBN [44,52]. The intrinsic radiative processes bypass the extrinsic nonradiative ones in $\mathrm{hBN}$, and they occur in a few tens of ps [63], because of the high oscillator strength due to electronic flat bands rather than because of the strong exciton-phonon coupling, as previously suggested [64]. Fifteen years after the pioneering work of Watanabe et al. [44], we thus unveil the physics behind the intense emission in $\mathrm{hBN}$.

Our results bridge the fundamental physics of flat bands with applications where the giant light-matter interaction emerges as a resource for improved performances in optoelectronic devices. Moreover, our work opens exciting perspectives in cavity quantum electrodynamics by showing the possibility to couple photonic modes to excitonic states with an ultralarge oscillator strength in systems with flat bands.

We gratefully acknowledge P. Vuong for fruitful discussions. This work and the Ph.D. funding of C. E. were financially supported by the network GaNeX (ANR-11LABX-0014) and the BONASPES project (ANR-19CE30-0007). J. L. and J.H.E. are grateful for support from the Office of Naval Research, Grant No. N0001420-1-2474, for the hBN crystal growth. This work was performed using HPC resources from GENCI-[TGCC/ CINES/IDRIS] within Project No. 100834.

*guillaume.cassabois@umontpellier.fr

[1] D. Leykam, A. Andreanov, and S. Flach, Adv. Phys. X 3, 1473052 (2018).

[2] Y. Cao et al., Nature (London) 556, 80 (2018).

[3] L. Balents, C. Dean, D. K. Efetov, and A. F. Young, Nat. Phys. 16, 725 (2020).

[4] G. Chen et al., Nature (London) 579, 56 (2020).

[5] K. F. Mak, C. Lee, J. Hone, J. Shan, and T. F. Heinz, Phys. Rev. Lett. 105, 136805 (2010).

[6] G. Wang, A. Chernikov, M. M. Glazov, T. F. Heinz, X. Marie, T. Amand, and B. Urbaszek, Rev. Mod. Phys. 90, 021001 (2018).

[7] G. Cassabois, P. Valvin, and B. Gil, Nat. Photonics 10, 262 (2016).

[8] C. Elias et al., Nat. Commun. 10, 2639 (2019).

[9] J. Caldwell, I. Aharonovich, G. Cassabois, J. H. Edgar, B. Gil, and D. N. Basov, Nat. Rev. Mater. 4, 552 (2019).

[10] B. Arnaud, S. Lebègue, P. Rabiller, and M. Alouani, Phys. Rev. Lett. 96, 026402 (2006).

[11] G. Fugallo, M. Aramini, J. Koskelo, K. Watanabe, T. Taniguchi, M. Hakala, S. Huotari, M. Gatti, and F. Sottile, Phys. Rev. B 92, 165122 (2015).

[12] F. Paleari, T. Galvani, H. Amara, F. Ducastelle, A. MolinaSánchez, and L. Wirtz, 2D Mater. 5, 045017 (2018).

[13] W. Hanke and L. J. Sham, Phys. Rev. Lett. 43, 387 (1979).

[14] See Supplemental Material at http://link.aps.org/ supplemental/10.1103/PhysRevLett.127.137401 for more information on ab initio calculations, reflectivity modelling and experimental details, which includes Refs. [15-39].

[15] G. Strinati, Riv. Nuovo Cimento 11, 1 (1988).

[16] L. Hedin, Phys. Rev. 139, A796 (1965).

[17] M. Gatti and F. Sottile, Phys. Rev. B 88, 155113 (2013).

[18] G. Onida, L. Reining, and A. Rubio, Rev. Mod. Phys. 74, 601 (2002).

[19] V. Olevano and L. Reining, Phys. Rev. Lett. 86, 5962 (2001).

[20] P. Hohenberg and W. Kohn, Phys. Rev. 136, B864 (1964).

[21] W. Kohn and L. J. Sham, Phys. Rev. 140, A1133 (1965).

[22] N. Troullier and J. L. Martins, Phys. Rev. B 43, 1993 (1991).

[23] X. Gonze, Z. Kristallogr. 220, 558 (2005).

[24] V. L. Solozhenko, G. Will, and F. Elf, Solid State Commun. 96, 1 (1995).

[25] H. Henck et al. Phys. Rev. B 95, 085410 (2017).

[26] A. Segura, L. Artús, R. Cuscó, T. Taniguchi, G. Cassabois, and B. Gil, Phys. Rev. Mater. 2, 024001 (2018).

[27] A. Marini and R. Del Sole, Phys. Rev. Lett. 91, 176402 (2003).

[28] K. Shindo, J. Phys. Soc. Jpn. 29, 287 (1970).

[29] F. Bechstedt, K. Tenelsen, B. Adolph, and R. Del Sole, Phys. Rev. Lett. 78, 1528 (1997).

[30] M. Rohlfing and S. G. Louie, Phys. Rev. B 62, 4927 (2000).

[31] G. Strinati, Phys. Rev. Lett. 49, 1519 (1982).

[32] G. Wang, X. Marie, I. Gerber, T. Amand, D. Lagarde, L. Bouet, M. Vidal, A. Balocchi, and B. Urbaszek, Phys. Rev. Lett. 114, 097403 (2015). 
[33] L. Sponza, H. Amara, F. Ducastelle, A. Loiseau, and C. Attaccalite, Phys. Rev. B 97, 075121 (2018).

[34] G. Cassabois, P. Valvin, and B. Gil, Phys. Rev. B 93, 035207 (2016).

[35] F. Paleari, H. P. C. Miranda, A. Molina-Sánchez, and L. Wirtz, Phys. Rev. Lett. 122, 187401 (2019).

[36] T. Q. P. Vuong, G. Cassabois, P. Valvin, V. Jacques, R. Cuscó, L. Artús, and B. Gil, Phys. Rev. B 95, 045207 (2017).

[37] P. Valvin, T. Pelini, G. Cassabois, A. Zobelli, J. Li, J. H. Edgar, and B. Gil, AIP Adv. 10, 075025 (2020).

[38] S. Liu, R. He, Z. Ye, X. Du, J. Lin, H. Jiang, B. Liu, and J. H. Edgar, Cryst. Growth Des. 17, 4932 (2017).

[39] J. Li et al., J. Mater. Chem. C 8, 9931 (2020).

[40] Interacting Electrons: Theory and Computational Approaches, edited by R. M. Martin, L. Reining, and D. M. Ceperley (Cambridge University Press, Cambridge, England, 2016).

[41] J. Koskelo, G. Fugallo, M. Hakala, M. Gatti, F. Sottile, and P. Cudazzo, Phys. Rev. B 95, 035125 (2017).

[42] S. Galambosi et al., Phys. Rev. B 83, 081413(R) (2011).

[43] D. M. Hoffman, G. L. Doll, and P. C. Eklund, Phys. Rev. B 30, 6051 (1984).

[44] K. Watanabe, T. Taniguchi, and H. Kanda, Nat. Mater. 3, 404 (2004).

[45] K. Watanabe and T. Taniguchi, Phys. Rev. B 79, 193104 (2009).

[46] Fundamentals of Semiconductors, edited by P. Y. Yu and M. Cardona (Springer-Verlag, Berlin Heidelberg, 1996).

[47] T. Q. P. Vuong, G. Cassabois, P. Valvin, V. Jacques, A. Van Der Lee, A. Zobelli, K. Watanabe, T. Taniguchi, and B. Gil, 2D Mater. 4, 011004 (2017).
[48] P. J. Dean and G. G. Thomas, Phys. Rev. 150, 690 (1966).

[49] Y. Kaifu and T. Komatsu, J. Phys. Soc. Jpn. 40, 1377 (1976).

[50] R. Ishii et al., Jpn. J. Appl. Phys. 59, 010903 (2020).

[51] D. Auvergne, P. Merle, and H. Mathieu, Phys. Rev. B 12, 1371 (1975).

[52] L. Schué et al., Phys. Rev. Lett. 122, 067401 (2019).

[53] Semiconductor Optics, edited by C.F. Klingshirn (Springer-Verlag, Berlin Heidelberg, 2007).

[54] M. M. Denisov and V. P. Makarov, Phys. Status Solidi (b) 56, 9 (1973).

[55] L. C. Andreani, F. Bassani, and A. Quattropani, Nuovo Cimento D 10, 1473 (1988).

[56] Physics of Wurtzite Nitrides and Oxides, Springer Series in Materials Science, edited by B. Gil (Springer International Publishing, Switzerland, 2014).

[57] F. Beerwerth and D. Fröhlich, Phys. Rev. Lett. 55, 2603 (1985).

[58] F. Beerwerth, D. Fröhlich, P. Köhler, V. Leinweber, and A. Voss, Phys. Rev. B 38, 4250 (1988).

[59] S. Galamić-Mulaomerović and C. H. Patterson, Phys. Rev. B 72, 035127 (2005).

[60] R. J. Elliott, Phys. Rev. 108, 1384 (1957).

[61] Y. Nanishi, Nat. Photonics 8, 884 (2014).

[62] M. Kneissl, T.-Y. Seong, J. Han, and H. Amano, Nat. Photonics 13, 233 (2019).

[63] S. F. Chichibu, Y. Ishikawa, H. Kominami, and K. Hara, J. Appl. Phys. 123, 065104 (2018).

[64] T. Q. P. Vuong, G. Cassabois, P. Valvin, S. Liu, J. H. Edgar, and B. Gil, Phys. Rev. B 95, 201202(R) (2017). 